\title{
Robot-assisted thoracic surgery in Colombia: a multi-institutional initial experience
}

\author{
Miguel Ricardo Buitrago ${ }^{1,2,3,4}$, Juliana Restrepo ${ }^{5}$ \\ ${ }^{1}$ Department of Thoracic Surgery, Instituto Nacional de Carcerología, Universidad Militar Nueva Granada, Bogotá, Colombia; ${ }^{2}$ Department of \\ Thoracic Surgery, Clínica de Marly, Bogotá, Colombia; ${ }^{3}$ Department of Thoracic Surgery, Clínica Shaio, Bogotá, Colombia; ${ }^{4}$ Thoracic Surgery, El \\ Bosque University, Bogotá, Colombia; ${ }^{5}$ Department of Surgical Oncology, Instituto Nacional de Cancerología, Universidad Militar Nueva Granada, \\ Bogotá, Colombia \\ Correspondence to: Miguel Ricardo Buitrago. Clínica de tórax, Instituto Nacional de Cancerología, Universidad Militar Nueva Granada, Calle 1 No. 9, \\ 85, Bogotá, Colombia. Email: buitrago77us@yahoo.com.
}

Background: Robotic assisted videothoracoscopic surgery (RVATS) adoption has increased worldwide from $3.4 \%$ in 2010 to $17.5 \%$ in 2015 . However, in Latin America, the literature is limited to a report of a series of 10 patients who underwent RVATS lobectomy and one case report of an RVATS thymectomy from Brazil.

Methods: This is a retrospective review of all RVATS performed in Bogotá Colombia since 2012. A single thoracic surgeon (RB) performed all the operations at three institutions: Clínica de Marly, Fundación Clínica Shaio and Instituto Nacional de Cancerología. Preoperative, intraoperative, postoperative and pathology report variables were included. Patients were analyzed in three groups: robotic RVATS pulmonary resections, RVATS mediastinal surgeries and other RVATS procedures. Descriptive statistics were used to report the median and interquartile range (IQR) of the continuous variables, and number and percentage were used to describe categorical variables. The association between total operative time and the year the surgery was analyzed using a linear regression model.

Results: Forty-seven patients underwent RVATS pulmonary resections; $72.3 \%(\mathrm{n}=34)$ of these patients underwent a RVATS lobectomy. The median total operative time was 220 (IQR: 200 to 250) minutes, 6.4\% $(\mathrm{n}=3)$ had intraoperative complications, and the most frequent histologic diagnosis was adenocarcinoma $(\mathrm{n}=24,51.1 \%)$. Of 18 patients who underwent RVATS mediastinal surgeries, $50.0 \%(\mathrm{n}=9)$ had RVATS thymectomy, the median total operative time was 195.5 (IQR: 131 to 221 ) minutes and two patients (11.1\%) had intraoperative complications. The linear regression model of the association between total operative time and the year the surgery showed a 10.3 minute reduction per year $(\mathrm{P}=0.006)$.

Conclusions: This is the second series of RVATS published in Latin America and the first published in Colombia, with comparable perioperative results to other reports.

Keywords: Robot-assisted thoracoscopic surgery (RATS); video-assisted thoracoscopic surgery (VATS); da Vinci surgical system; thoracic surgery; pulmonary adenocarcinoma

Submitted Nov 26, 2018. Accepted for publication Feb 08, 2019.

doi: 10.21037/acs.2019.03.01

View this article at: http://dx.doi.org/10.21037/acs.2019.03.01

\section{Introduction}

Minimally invasive surgery (MIS) has demonstrated substantial benefits compared with thoracotomy and sternotomy for thoracic procedures. These benefits include lower complication rates, shorter hospital length of stay (LOS) and faster recovery. Robotic-assisted videothoracoscopic surgery (RVATS) is the most recent MIS technique and compared with other MIS platforms, RVATS provides three dimensional visualization, seven 
degrees of freedom motion and improved visual haptics (1).

In 2015 , more than 6,000 robotic lobectomies were performed in the United States, and more than 8,600 were done worldwide (2). In contrast, the Latin American literature is scarce: a RVATS pulmonary lobectomy series from Brazil with 10 patients and good perioperative outcomes has been published but long term results are pending (3). Similarly, one case report of robotic assisted thymectomy also from Brazil has been published (4). In Colombia, the first robotic lobectomy was performed in 2012 (5), when the da Vinci Robotic System was acquired. Since then, 69 RVATS have been performed.

The objective of this article is to report the first experience with RVATS for different thoracic pathologies published in Colombia and present the largest series published in Latin America.

\section{Methods}

This is a retrospective review of all RVATS performed in Bogotá, Colombia since 2012 (when the first da Vinci robot was acquired). A single thoracic surgeon (RB) performed all the operations at three institutions: Clínica Marly, Clínica Shaio and Instituto Nacional de Cancerología. The operations were completed with the patients under general anesthesia and lung isolation, using either a 3-arm or 4-arm robotic technique. All the patients who underwent RVATS from April 2012 to August 2018 were selected. The clinical histories, surgical descriptions and pathology results were reviewed and the variables were collected in a pre-design format.

Preoperative variables included age and sex. Intraoperative variables included lesion location and resection type; docking time, console time and total operative time in minutes (min). The docking time was defined as time from the first skin incision to the start of driving the robotic arm while seated at the console, the console time was defined as the time that principal surgeon drove the robotic arm while seated at the console and performed the intrathoracic procedures and total operative time was measured from the first skin incision to skin closure. Blood loss in milliliters $(\mathrm{mL})$, conversion rate to thoracotomy or sternotomy and intraoperative complications were also measured. Postoperative variables collected included chest tube duration in hours (chest tubes were removed during the hospital stay as soon as the drainage was less than $100 \mathrm{~mL} /$ day and no air leaks were present), postoperative complications, hospital length-of-stay (days), in hospital mortality, 90 day mortality and follow-up time (months). Variables collected from pathology reports included histology of the tumor or lesion, median tumor size in millimeters $(\mathrm{mm})$ and the stage of the disease for malignant pathologies (complemented with images and clinical history).

Patients were divided in three groups: patients who were subject to robotic RVATS pulmonary resections, patients who were subject to RVATS mediastinal surgeries and patients who were subject to other RVATS procedures which include plication of the diaphragm, sympathectomy and resection of a thoracic wall lesion. The variables were analyzed in each group separately.

The data were collected in Excel (Microsoft Corp) and analyzed using STATA 14 (Stata Corp LLC). Descriptive statistics were used to report the median and interquartile range (IQR) of continuous variables (with 25 th and 75 th centiles given as the IQR). Number and percentage were used to describe categorical variables. The association between total operative time and the year the surgery was performed was analyzed using a linear regression model.

\section{Results}

\section{RVATS pulmonary resections}

Between April 2012 and August 2018, a total of 69 patients underwent RVATS with the da Vinci Surgical System. From the 47 patients in which RVATS pulmonary resections were performed, the median age was 61 (IQR: 50 to 73$)$ years and $27.7 \%(n=13)$ were men. The data for this group is presented in Table 1. Tumor location was most commonly the right upper lobe $(\mathrm{n}=15,31.9 \%) .72 .3 \%$ $(n=34)$ of patients underwent a RVATS lobectomy and the remainder had segmental $(\mathrm{n}=2,4.3 \%)$ or non anatomic resections $(\mathrm{n}=5,10.6 \%)$. Two bronchoplasties were performed for two patients with a neuroendocrine tumor and a metastatic lesion from thyroid cancer. The median total operative time was 220 (IQR: 200 to 250) minutes, the median console time was 125 (IQR: 110 to 150 ) minutes and the median blood loss was 100 (IQR: 80 to 100) $\mathrm{mL}$. There were no conversions to thoracotomy and $6.4 \%(n=3)$ had intraoperative complications (intraoperative bleeding that was adequately controlled). The median chest tube duration was 48 (IQR: 48 to 67 ) hours and the median length of hospital stay was 3 (IQR: 2 to 4 ) days. The most frequent histologic diagnosis was adenocarcinoma $(n=24$, $51.1 \%)$, followed by benign lesions $(\mathrm{n}=9,19.2 \%)$ and 
Table 1 RVATS pulmonary resections: patient characteristics and perioperative results

\begin{tabular}{ll}
\hline Preoperative variables $(\mathrm{n}=47)$ & $\mathrm{N}(\%)$ or median (IQR) \\
\hline Age (years) & $61(50$ to 73$)$ \\
Male & $13(27.7)$
\end{tabular}

Intraoperative variables

Lesion location

Right upper lobe

$15(31.9)$

Right middle lobe

7 (14.9)

Right lower lobe

$11(23.4)$

Left upper lobe

$8(17.0)$

Left lower lobe

6 (12.8)

Resection type

Lobectomy

34 (72.3)

Segmental resection

2 (4.3)

Non anatomic resection (wedge)

5 (10.6)

Lobectomy and wedge

3 (6.4)

Lobectomy and pleural biopsy

$1(2.1)$

Lobectomy and bronchoplasty

$2(4.3)$

Docking time (min)

18 (13 to 20$)$

Console time ( $\mathrm{min})$

125 (110 to 150$)$

Total operative time (min)

220 (200 to 250 )

Blood loss $(m L)$ ( $n=46,1$ missing)

100 (80 to 100$)$

Conversion

0 (0)

Intraoperative complications

3 (6.4)

Postoperative variables $(n=47)$

Chest tube duration (hours)

48 (48 to 67$)$

Postoperative complications

5 (10.6)

Cardiac arrhythmia

$2(4.3)$

Air leak

2 (4.3)

Pneumonia

$1(2.1)$

Hospital length of stay (days)

3 (2 to 4$)$

In-hospital mortality

$0(0)$

90 day mortality ( $n=40,7$ missing)

1 (2.5)

Follow-up time (months)

29 (12 to 55$)$

Table 1 (continued)

\begin{tabular}{ll} 
Table 1 (continued) & $\mathrm{N}(\%)$ or median (IQR) \\
\hline Preoperative variables $(\mathrm{n}=47)$ & $10(10$ to 20$)$ \\
\hline $\begin{array}{l}\text { Histology result variables }(\mathrm{n}=47) \\
\text { Tumor size }(\mathrm{mm})(\mathrm{n}=38,9 \text { benign } \\
\text { disease) }\end{array}$ & \\
Lesion histology & $24(51.1)$ \\
Adenocarcinoma & $3(6.4)$ \\
Squamous cell carcinoma & $5(10.6)$ \\
Neuroendocrine tumor & $2(4.3)$ \\
Large cell carcinoma & $4(8.5)$ \\
Metastatic disease & $9(19.1)$ \\
Benign & $3(18.9)$ \\
Pathologic stage ( $\mathrm{n}=37,9$ benign, 1 missing value) \\
I
\end{tabular}

Continuous variables are expressed as median and interquartile range (25\% quartile to $75 \%$ quartile). Categorical variables are expressed as count and percentage. RVATS, robotic assisted videothoracoscopic surgery.

neuroendocrine tumors $(\mathrm{n}=5,10.6 \%)$. The majority of patients had stage I disease $(\mathrm{n}=24,64.9 \%)$. There was no in-hospital mortality and there was one death at 90 days follow-up. The mean follow-up time was 29 (IQR: 12 to 55) months and during the follow-up time, $6(12.8 \%)$ patients died of metastatic disease. Two patients with marginal pulmonary function (FEV1 49\% and 26\%) underwent a RVATS lobectomy with good results.

\section{RVATS mediastinal procedures}

There were 18 patients who underwent RVATS mediastinal surgeries. In this group the median age was 50 (IQR: 39 to 59$)$ years and $22.2 \%(n=4)$ were men. The data for this group is presented in Table 2. The surgical site was most commonly the anterior mediastinum $(\mathrm{n}=11,61.1 \%)$. Among them, 50.0\% ( $n=9)$ of patients underwent a RVATS thymectomy, seven patients had myasthenia gravis and two patients had thymomas. There were two esophageal 
Table 2 RVATS mediastinal lesion resection: patient characteristics and perioperative results

\begin{tabular}{|c|c|}
\hline Preoperative variables $(n=18)$ & $\begin{array}{l}\text { No. }(\%) \text { or median } \\
\text { (IQR) }\end{array}$ \\
\hline Age (years) & 50 (39 to 59$)$ \\
\hline Male & $4(22.2)$ \\
\hline \multicolumn{2}{|l|}{ Intraoperative variables $(n=18)$} \\
\hline \multicolumn{2}{|l|}{ Lesion location } \\
\hline Anterior mediastinum & $11(61.1)$ \\
\hline Middle mediastinum & $6(33.3)$ \\
\hline Posterior mediastinum & $1(5.6)$ \\
\hline \multicolumn{2}{|l|}{ Resection type } \\
\hline Thymectomy & $9(50.0)$ \\
\hline Other mediastinal mass resection & $5(27.8)$ \\
\hline Esophageal lesion resection & $2(11.1)$ \\
\hline Mediastinal biopsy & $1(5.6)$ \\
\hline Bronchogenic cyst resection & $1(5.6)$ \\
\hline Docking time (min) & 15 (10 to 20$)$ \\
\hline Console time (min) & 107.5 (95 to 125$)$ \\
\hline Total operative time (min) & 195.5 (131 to 221$)$ \\
\hline Blood loss (mL) & 50 (20 to 90$)$ \\
\hline Conversion & $0(0)$ \\
\hline Intraoperative complications & $2(11.1)$ \\
\hline \multicolumn{2}{|l|}{ Postoperative variables $(n=18)$} \\
\hline Chest tube duration (hours) & 48 (24 to 68$)$ \\
\hline Postoperative complications & $0(0)$ \\
\hline Length of hospital stay (days) & 2.5 (2 to 3 ) \\
\hline In-hospital mortality & $0(0)$ \\
\hline 90-day mortality ( $\mathrm{n}=16,2$ missing) & $0(0)$ \\
\hline Follow-up time (months) & 14 (5 to 24$)$ \\
\hline \multicolumn{2}{|l|}{ Histology variables $(n=18)$} \\
\hline Tumor size $(\mathrm{mm})(\mathrm{n}=16,2$ missing) & 35 (25 to 50$)$ \\
\hline \multicolumn{2}{|l|}{ Lesion histology } \\
\hline Thymic hyperplasia & 7 (38.9) \\
\hline Thymoma & $2(11.1)$ \\
\hline Metastatic disease & $3(16.7)$ \\
\hline Esophageal leiomyoma & $2(11.1)$ \\
\hline Parathyroid adenoma & $1(5.6)$ \\
\hline Shwannoma & $1(5.6)$ \\
\hline Intrathoracic goiter & $1(5.6)$ \\
\hline Bronchogenic cyst & $1(5.6)$ \\
\hline
\end{tabular}

Continuous variables are expressed as median and interquartile range ( $25 \%$ quartile to $74 \%$ quartile). Categorical variables are expressed as count and percentage. RVATS, robotic assisted videothoracoscopic surgery. leiomyoma resections and one bronchogenic cyst resection. The median total operative time was 195.5 (IQR: 131 to 221) minutes, the median console time was 107.5 (IQR: 95 to 125 ) minutes and the median blood loss was 50 (IQR: 20 to 90$) \mathrm{mL}$. There were no conversions to sternotomy and two patients $(11.1 \%)$ had intraoperative complications caused by bleeding. The median length of hospital stay was 2.5 (IQR: 2 to 3) days and the median chest tube duration was 48 (IQR: 24 to 68 ) hours. There were no in-hospital mortalities or 90-day mortalities and the mean follow-up time was 14 (IQR: 5 to 24) months. There were no deaths during the follow-up time.

\section{Other RVATS procedures}

There were four patients who underwent other types of surgery, consisting of two plicatures of the diaphragm, one sympathectomy and one thoracic wall resection of a patient who had a metastatic lesion from multiple myeloma (Table 3). There were no conversions, intraoperative complications, postoperative complications, in-hospital mortalities or 90-day mortality among these patients.

\section{Time trend for surgery time}

We performed a linear regression to analyze the association between total operative time and the year the surgery was performed (Figure 1). This figure shows a 10.3 minute reduction per year of surgery ( $95 \%$ confidence interval: 3.1-17.5 minutes, $\mathrm{P}=0.006$ ).

There were seven patients who had missing data on inhospital mortality because they were either lost to follow-up or had not completed the 90 days of follow-up at the time of data collection. Additionally, one patient had missing data on blood loss.

\section{Discussion}

In 2015, RVATS lobectomies accounted for $17.5 \%$ of elective lobectomies (6) - this represents a fivefold increase in the RVATS adoption rate [reported as 3.4\% of lobectomies in 2014 by Kent and colleagues (7)]. The increased adoption of RVATS appears to result from a shift from the open approach, given that the rate of video assisted thoracoscopic surgeries (VATS) have remained unchanged at approximately $40 \%$ (6). The reasons for this limited acceptance of VATS are multifactorial and 


\begin{tabular}{|c|c|c|c|c|}
\hline Type of surgery & Plicature of diaphragm & Plicature of diaphragm & Sympathectomy & Thoracic wall lesion resection \\
\hline Age (years) & 34 & 60 & 34 & 48 \\
\hline Sex & Male & Female & Female & Female \\
\hline Docking time (min) & 20 & 27 & 16 & 15 \\
\hline Console time (min) & 70 & 120 & 20 & 90 \\
\hline Total operative time (min) & 148 & 224 & 82 & 176 \\
\hline Blood loss (mL) & 90 & 50 & 20 & 90 \\
\hline
\end{tabular}

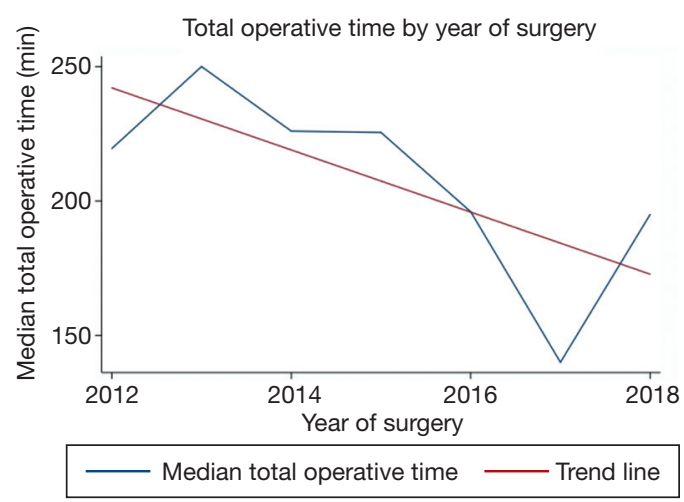

Figure 1 Linear regression showing a 10.3 minute reduction in total operative time by years of RVATS experience. RVATS, robotic assisted videothoracoscopic surgery.

include restricted vision secondary to the two-dimensional nature of conventional laparoscopes, and limited range of motion of instruments due to size and design. RVATS has been studied to overcome these limitations because it offers several technical advantages such as threedimensional, high definition field of view, tremor dampening, augmented dexterity, and better ergonomics (8-11). Patients undergoing VATS suffer from fewer complications, have less pain and blood loss, and recover faster compared to patients undergoing thoracotomy for different thoracic pathologies (8). In multivariate analysis, VATS was associated independently with a reduced risk of complications (12). Several retrospective comparative studies published between 2008 and 2018 have demonstrated that RVATS lobectomy is safe and effective and has 30-day mortality comparable to that of VATS (13-16) and better outcomes compared to thoracotomy $(6,7,17)$, with similar long term survival $(18,19)$. When single large national and statewide databases are analyzed, RVATS yields lower morbidity (6) and lower mortality than both thoracotomy and VATS $(7,17)$. Two studies found lower conversion rates with RVATS compared to VATS $(15,16)$ and some studies suggest that RVATS has better outcomes in patients with marginal pulmonary function (20) and that it is safe for older patients (21). In our series, two patients with marginal pulmonary function had RVATS lobectomy with good results. Some systematic reviews and meta-analysis of retrospective observational studies found that perioperative morbidity and mortality were similar between patients who underwent lung resections by RVATS and those who underwent VATS $(9,10,12)$, with a tendency towards shorter hospitalization time and drainage duration with RVATS (8).

In this series, we present the first report of RVATS in Colombia and the second and largest series reported so far in Latin America. RVATS lobectomies were performed using the method described by Dylewski (22) and the instrument position is shown in Figure 2. Compared to the first series reported in Brasil, we have a higher female rate and a similar age distribution but we can not compare the perioperative results because they did not report median values of these variables. Most of their patients 


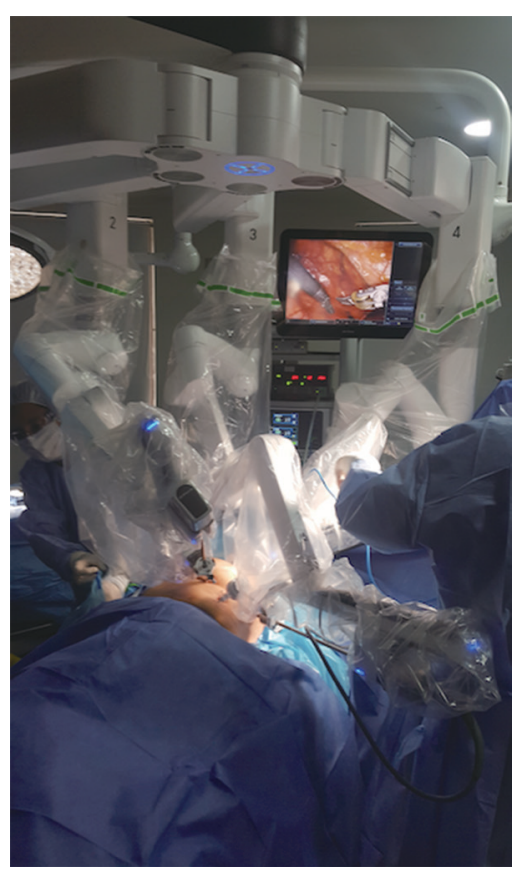

Figure 2 Instrument position for a RVATS pulmonary lobectomy with a three arm surgical technique. RVATS, robotic assisted videothoracoscopic surgery.

had chest tube drainage from 24 to 48 hours and length of hospital stay less than 48 hours, which concur with our results (3). Our results are comparable to those reported in the international literature, with the exception of sex distribution because our series has more females compared to others (10). Our total operative time is also comparable: the mean operative time in our study was $185 \mathrm{~min}$ and in the literature it ranges from 107 to 241 minutes $(9,10)$. As we know, there is a steep learning curve for RVATS, and operating time has been shown to significantly improve after the initial learning period $(23,24)$. In this report we analyzed the total operative time by the year in which the surgical procedures were performed and the mean total time decreased during the six years of the study (Figure 1). Conversion rates of $0 \%$ were reported in many series $(25,26)$. Postoperative hospital LOS, duration of chest tube drainage, perioperative mortality, perioperative morbidity and blood loss in this series were comparable to those previously reported $(9,10,27)$.

Most surgeons offered RVATS to clinical stage I or II non small cell lung cancer: Veronesi et al. limited the maximum size to five centimeters, Cerfolio et al. extended the indications to include larger size or preoperative chemoradiation and other surgeons allowed more advanced cases (28). Recent reports showed that more complicated cases needing bronchoplastic surgery were feasible for robotic surgery $(29,30)$. In this series most of the patients with a lung cancer diagnosis had stage I disease and we also report more complex surgical procedures such as two bronchoplasties with adequate perioperative outcomes.

Mediastinal tumor patients are good candidates for robotic surgery; several articles have shown that RVATS mediastinal mass resections including thymectomy are as good as VATS (31) and superior to transsternal resection, reducing intraoperative blood loss, postoperative complications, and hospital LOS (32-34). RVATS for thymic epithelial tumors achieved comparable longterm oncologic outcomes (35). There are two metaanalysis $(36,37)$ and one systematic review (38) comparing RVATS and VATS for thymectomy-these show no difference with respect to conversion rate, hospital LOS, or postoperative morbidity. One meta-analysis showed a tendency towards reduced hospital LOS and reduced chest tube drainage days with RVATS compared to VATS but the power of the study was low [only seven studies were included (37)].

This series of RVATS for mediastinal surgeries has similar results compared with the literature. In the metaanalysis by Buentzel et al., the operation time ranged from 97 to 224.2 minutes and in our study the mean total operative time was in this range (37). As in this series, most of the studies had $0 \%$ conversion rate, and short length of chest tube duration (37). The hospital LOS in the literature ranged from 3.7 to 9.6 days. In this series this time was shorter, with a mean LOS of 2.5 days (37). Meta-analysis showed blood loss was minimal with no postoperative complications (38). In the current report patients had minimal blood loss and no postoperative complications, similar to other reports in the literature $(39,40)$.

In conclusion, this series brings to the thoracic robotic literature the second series of RVATS published in Latin America and the first published in Colombia, with comparable perioperative results to other reports. We also present two patients who underwent RVATS bronchoplasties, showing that more complex procedures are feasible. Two patients with marginal pulmonary function that made the open approach impossible and the VATS approach very difficult are included, and in these patients, RVATS precision and post-operative outcomes made the pulmonary resection possible and ensured a good postoperative course. 


\section{Acknowledgements}

None.

\section{Footnote}

Conflicts of Interest: The authors have no conflicts of interest to declare.

\section{References}

1. Fahim C, Hanna W, Waddell T, et al. Robotic-Assisted thoracoscopic surgery for lung resection: The first Canadian series. Can J Surg 2017;60:260-5.

2. Wei B, Cerfolio RJ. Robotic Lobectomy and Segmentectomy: Technical Details and Results. Surg Clin North Am 2017;97:771-82.

3. Terra RM, Araujo PH, Lauricella LL, et al. Robotic pulmonary lobectomy for lung cancer treatment: program implementation and initial experience. J Bras Pneumol 2016;42:185-90.

4. da Silva RA, Zugai R, Bruno IR. Letter to the Editor: robotic thymectomy for myasthenia gravis. J Bras Pneumol 2011;37:694-6.

5. Buitrago MR, Franco A. Lobectomía pulmonar toracoscópica asistida por robot. Rev Colomb Cir 2012;27:65.

6. Oh DS, Reddy RM, Gorrepati ML, et al. Robotic-Assisted, Video-Assisted Thoracoscopic and Open Lobectomy: Propensity-Matched Analysis of Recent Premier Data. Ann Thorac Surg 2017;104:1733-40.

7. Kent M, Wang T, Whyte R, et al. Open, video-assisted thoracic surgery, and robotic lobectomy: Review of a national database. Ann Thorac Surg 2014;97:236-42.

8. Emmert A, Straube C, Buentzel J, et al. Robotic versus thoracoscopic lung resection: A systematic review and meta-analysis. Medicine (Baltimore) 2017;96:e7633.

9. Agzarian J, Fahim C, Shargall Y, et al. The Use of Robotic-Assisted Thoracic Surgery for Lung Resection: A Comprehensive Systematic Review. Semin Thorac Cardiovasc Surg 2016;28:182-92.

10. Cao C, Manganas C, Ang SC, et al. A systematic review and meta-analysis on pulmonary resections by robotic video-assisted thoracic surgery. Ann Cardiothorac Surg 2012;1:3-10.

11. Radkani $\mathrm{P}$, Joshi $\mathrm{D}$, Barot $\mathrm{T}$, et al. Robotic videoassisted thoracoscopic lung resection for lung tumors: a community tertiary care center experience over four years.
Surg Endosc 2016;30:619-24.

12. Ye X, Xie L, Chen G, et al. Robotic thoracic surgery versus video-assisted thoracic surgery for lung cancer: A metaanalysis. Interact Cardiovasc Thorac Surg 2015;21:409-14.

13. Louie BE, Wilson JL, Kim S, et al. Comparison of VideoAssisted Thoracoscopic Surgery and Robotic Approaches for Clinical Stage I and Stage II Non-Small Cell Lung Cancer Using The Society of Thoracic Surgeons Database. Ann Thorac Surg 2016;102:917-24.

14. Adams RD, Bolton WD, Stephenson JE, et al. Initial multicenter community robotic lobectomy experience: Comparisons to a national database. Ann Thorac Surg 2014;97:1893-8.

15. Mungo B, Hooker CM, Ho JSY, et al. Robotic Versus Thoracoscopic Resection for Lung Cancer: Early Results of a New Robotic Program. J Laparoendosc Adv Surg Tech A 2016;26:243-8.

16. Mahieu J, Rinieri P, Bubenheim M, et al. RobotAssisted Thoracoscopic Surgery versus Video-Assisted Thoracoscopic Surgery for Lung Lobectomy Can a Robotic Approach Improve Short-Term Outcomes and Operative Safety [abstract] - Mahieu. Thorac Cardiovasc Surg 2016;64:354-62.

17. Farivar AS, Cerfolio RJ, Vallières E, et al. Comparing robotic lung resection with thoracotomy and videoassisted thoracoscopic surgery cases entered into the society of thoracic surgeons database. Innovations (Phila) 2014;9:10-5.

18. Yang HX, Woo KM, Sima CS, et al. Long-term survival based on the surgical approach to lobectomy for clinical stage i nonsmall cell lung cancer: Comparison of robotic, video-assisted thoracic surgery, and thoracotomy lobectomy. Ann Surg 2017;265:431-7.

19. Park BJ. Robotic Lobectomy for Non-Small Cell Lung Cancer. Thorac Surg Clin 2014;24:157-62.

20. Kneuertz PJ, D'Souza DM, Moffatt-Bruce SD, et al. Robotic lobectomy has the greatest benefit in patients with marginal pulmonary function. J Cardiothorac Surg 2018;13:56.

21. Kass KS, Velez-Cubian FO, Zhang WW, et al. Effect of advanced age on peri-operative outcomes after roboticassisted pulmonary lobectomy: Retrospective analysis of 287 consecutive cases. J Geriatr Oncol 2017;8:102-7.

22. Dylewski MR, Ohaeto AC, Pereira JF. Pulmonary Resection Using a Total Endoscopic Robotic VideoAssisted Approach. Semin Thorac Cardiovasc Surg 2011;23:36-42.

23. Melfi FMA, Mussi A. Robotically Assisted Lobectomy: 
Learning Curve and Complications. Thorac Surg Clin 2008;18:289-95.

24. Veronesi G. Robotic thoracic surgery technical considerations and learning curve for pulmonary resection. Thorac Surg Clin 2014;24:135-41.

25. Jang HJ, Lee HS, Park SY, et al. Comparison of the Early Robot-Assisted Lobectomy Experience to VideoAssisted Thoracic Surgery Lobectomy for Lung Cancer. Innovations 2011;6:305-10.

26. Anderson CA, Hellan M, Falebella A, et al. Roboticassisted lung resection for malignant disease. Innovations (Phila) 2007;2:254-8.

27. Velez-Cubian FO, Ng EP, Fontaine JP, et al. RoboticAssisted Videothoracoscopic Surgery of the Lung. Cancer Control 2015;22:314-25.

28. Yamashita S, Yoshida Y, Iwasaki A. Robotic surgery for thoracic disease. Ann Thorac Cardiovasc Surg 2016;22:1-5.

29. Yang SM, Kuo S, Lee J. Robot-assisted thoracoscopic bronchoplasty. J Vis Surg 2015;1:20.

30. Lazar JF, Posner DH, Palka W, et al. Robotically assisted bilateral bronchoplasty for tracheobronchomalacia. Innovations (Phila) 2015;10:428-30.

31. Jun Y, Hao L, Demin L, et al. Da Vinci robot-assisted system for thymectomy: experience of 55 patients in China. Int J Med Robot 2014;10:294.

32. Weksler B, Tavares J, Newhook TE, et al. Robot-assisted thymectomy is superior to transsternal thymectomy. Surg Endosc 2012;26:261-6.

Cite this article as: Buitrago MR, Restrepo J. Robot-assisted thoracic surgery in Colombia: a multi-institutional initial experience. Ann Cardiothorac Surg 2019;8(2):233-240. doi: 10.21037/acs.2019.03.01
33. Renaud S, Santelmo N, Renaud M, et al. Robotic-assisted thymectomy with da Vinci II versus sternotomy in the surgical treatment of non-thymomatous myasthenia gravis: Early results. Rev Neurol (Paris) 2013;169:30-6.

34. Seong YW, Kang CH, Choi JW, et al. Early clinical outcomes of robot-assisted surgery for anterior mediastinal mass: Its superiority over a conventional sternotomy approach evaluated by propensity score matching. Eur J Cardiothorac Surg 2014;45:e68-73.

35. Kang CH, Hwang Y, Lee HJ, et al. Robotic Thymectomy in Anterior Mediastinal Mass : Propensity Score Matching Study With. Ann Thorac Surg 2016;102:895-901.

36. Fok M, Bashir M, Harky A, et al. Video-Assisted Thoracoscopic Versus Robotic-Assisted Thoracoscopic Thymectomy: Systematic Review and Meta-analysis. Innovations (Phila) 2017;12:259-64.

37. Buentzel J, Straube C, Heinz J, et al. Thymectomy via open surgery or robotic video assisted thoracic surgery. Can a recommendation already be made? Medicine (Baltimore) 2017;96:e7161.

38. Buentzel J, Heinz J, Hinterthaner M, et al. Robotic versus thoracoscopic thymectomy: The current evidence. Int J Med Robot 2017;13:1-6.

39. Straughan DM, Fontaine JP, Toloza EM. Robotic-assisted videothoracoscopic mediastinal surgery. Cancer Control 2015;22:326-30.

40. Weissenbacher A, Bodner J. Robotic Surgery of the Mediastinum. Thorac Surg Clin 2010;20:331-9. 